\title{
Analysis of Chemometrics Date and Apportionment of Pollution Sources of Urban Rivers from Northeast China - a Case from Baitapu River
}

\author{
Bingfei Yan ${ }^{1, a}$, Jianfeng Peng ${ }^{2, b^{*}}$ \\ ${ }^{1}$ Department of Urban Water Environmental Research, Chinese Research Academy of \\ Environmental Sciences, Beijing 100012, China \\ ${ }^{2}$ College of Chemistry and Environmental Engineering, Shandong University of Science and \\ Technology, Qingdao 266590, China \\ aybf201506@163.com, bjif1995@163.com
}

\begin{abstract}
Keywords: Urban river; Spatial/temporal distribution; Principal component analysis (PCA); Source identification
\end{abstract}

Abstract. Baitapu River is an important tributary of Hun River and a typical city river in northeast China. Analysis of cheometrics date of water quality and environmental heterogeneity was used in 17 monitoring sites consisted of 12 water-quality parameters from 2012 to 2013. Spatial and temporal variations in water quality were assessed and pollution sources were identified which aimed to offer foundations to comprehensive treatment and the construction of water environment ecosystem for this kind of rivers in northeast China. Results of these monitoring indicators show that two segments is classified by spatial distribution, temporal distribution and Nemerow index: including upstream minor polluted segment in rural areas and downstream moderate polluted segment in urban areas. The main pollution of Baitapu River are TN, $\mathrm{NH}_{3}-\mathrm{N}, \mathrm{COD}, \mathrm{TOC}, \mathrm{TP}$, and chlorophyll a (Chl-a) based on principal component analysis (PCA). The upstream are mainly polluted by agricultural non-point pollution while the downstream are mainly contaminated by industrial wastewater and domestic sewage.

\section{Introduction}

City River water quality is one of the most important indicators for river ecological system[1]. However, river waters are most vulnerable to be polluted by wastewaters. Baitapu River is a tributary of Hun River basin and there exists lots of problems as much rivers of northeast china. Therefore, analysis of environmental heterogeneity and pollution sources of Baitapu River are of great significance. Multivariate statistical evaluation method as principal component analysis (PCA) and index evaluation method named Nemerow index were chosen to analyze distribution characteristics of water quality in Baitapu river[2,3]. Meanwhile, rely on the research on spatial distribution pattern of sampling point water quality and the simulation of spatial differentiation with kringing of spatial interpolation method, the present study aims to discover the differences in temporal and space based on the GIS platform and determine the sources and origins of Baitapu water contamination[4]. These conclusions aim to provide a certain amount of data to summarize and analyze the spatial and temporal distribution characteristic of pollutants and offer foundations to comprehensive treatment and the construction of water environment ecosystem for this kind of middle and small rivers in northeast china.

\section{Methods}

Study area

Baitapu river is located in the left side of the middle reaches of Hun river. It's an important tributary of Hun river and one of the main rivers in Shenyang. It originates Laotangyu village, LiXiang Town, Hunnan new district. Geographical coordinates is $41^{\circ} 38^{\prime} 10.8^{\prime \prime}$ latitude north and $123^{\circ} 39^{\prime} 6.7^{\prime \prime}$ longitude east. With a basin of $178 \mathrm{~km}^{2}$, the total length of the river is $48.5 \mathrm{~km}$, the mean stream gradient is $1.65 \%$ and the average annual runoff is $2790 \times 104 \mathrm{~m}^{3}$. 
Sampling sites and time

The data sets of Baitaipu river water quality from 17 monitoring sites consisted of 12 water-quality parameters. It was monitored 10 times over two years (September 2012, October 2012, November 2012, December 2012, February 2013, March 2013, April 2013, May 2013, July 2013, August 2013), (Fig. 1).

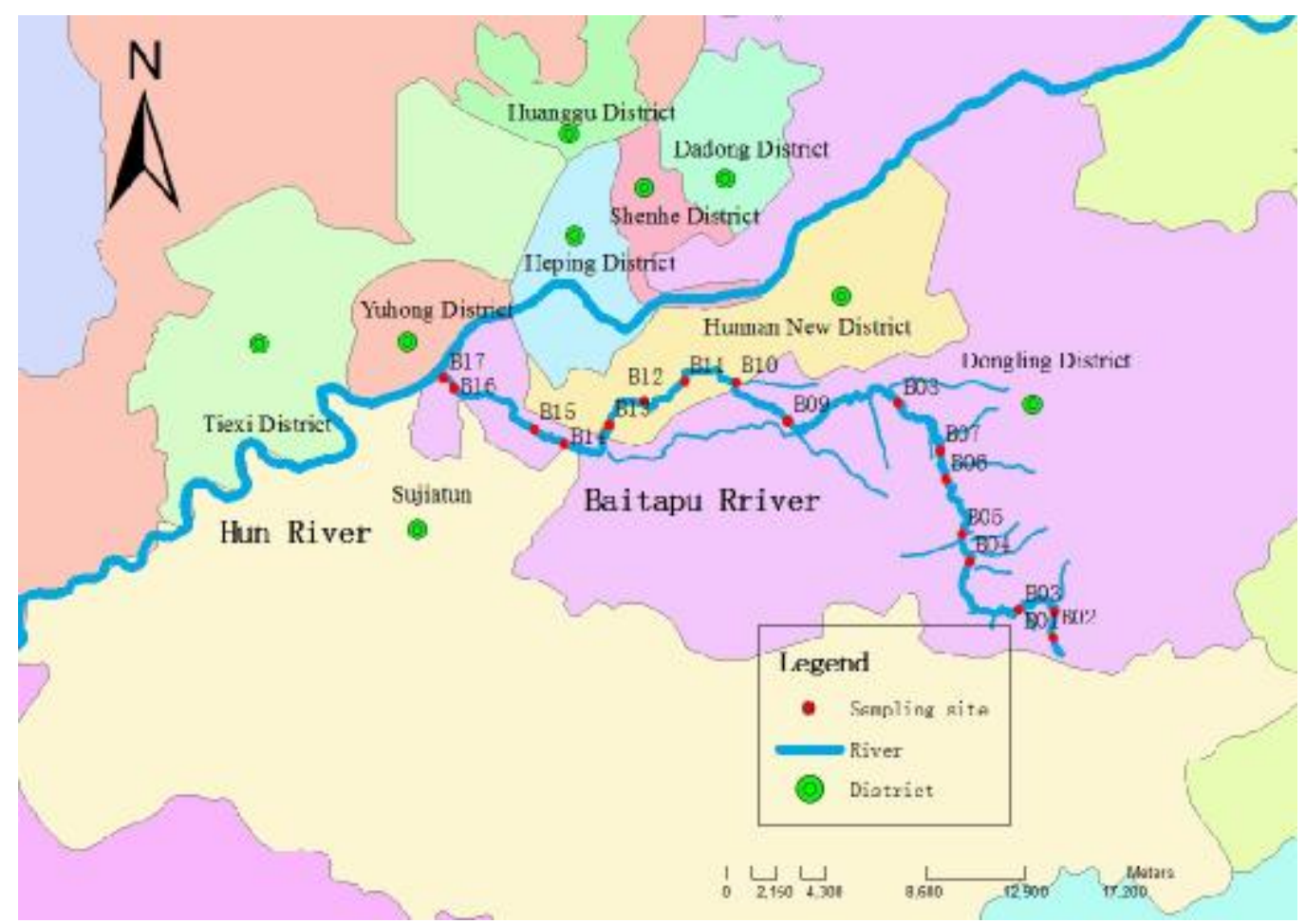

Sampling sites: B01-Source of the river; B02-Laotangyu ; B03-Xing village; B04-Lixiang;

B05-Lixiang new village; B06-Gaobazhai; B07-Yongan; B08-Shijiazhai ; B09-Yingchengzi;

B10-University of science and engineering; B11-The 21st century lake; B12-North of Baitapu; B13-West of Baitapu; B14-South street of Nanjing; B15-Shengli street; B16-Caozhongtun; B17-Hun mouth

Fig. 1 Sampling sites distribution

Sample collection and processing

Selected parameters included total nitrogen (TN), ammoniacal nitrogen $\left(\mathrm{NH}_{3}-\mathrm{N}\right)$, chemical oxygen demand(COD), total organic carbon (TOC), total phosphorus (TP), chlorophyll (Chl-a) and 5 day biochemical oxygen demand $\left(\mathrm{BOD}_{5}\right)$. Some of them, such as the temperature, DO, $\mathrm{pH}, \mathrm{ORP}$ and EC were in situ tested by multifunctional water quality analyzer. For the other parameters, the samples were taken back by portable incubator at low temperature.

Data analysis

Spatial interpolation is a method of inferring data of other unknown points by those known points in the same area[5]. In this study, Kriging interpolation was used to process 6 water quality indexes of 17 samples with arcmap 9.3 .

On the premise of no losing or as less as possible loss of original informations, the function of PCA is to convert multi-index into fewer new indicators[6,7]. The major pollutant informations of Baitapu river were included in these parameters and they were independent of each other. Meanwhile, the main state of river pollution can be reflected.

Nemerow index is one of the most common method to calculate comprehensive pollution index, which can represent the quality and the scale of water environment pollution in Baitapu river . 


\section{Results and discussion}

Spatial and temporal distribution of parameter means

Spatial distribution

Spatial distribution of monthly arithmetic mean values of 6 parameters can be obtained using a

GIS-based kriging approach and the results were manifested in fig.2. Regular indicators such as TN, $\mathrm{NH}_{3}-\mathrm{N}$, and TP, all present a steadily increasing trend from upstream to downstream. In comparison with the upper river, the results suggest that nitrogen and phosphorus pollution is more serious in the lower reaches. Another two indexes, COD and TOC both represent the organic pollution. The mean values of COD ranged from 20.83 to 50.67 [mg/L], indicating that the content of organic in middle reaches is higher and slightly decreased in downstream . The average load of Chl-a demonstrates a gradually rising tendency in the first half of the river and slight declines in the lower reaches. The possible reason is that the content of pollutant which including nitrogen and phosphors is relatively high, but the deterioration of water quality is not suitable for the growth of algae. According to all of features above, $\mathrm{TN}, \mathrm{NH}_{3}-\mathrm{N}, \mathrm{COD}$, TOC, TP and Chl-a are seriously exceeding the surface water quality levels of class $\mathrm{V}$ in some sections of the river. Other good many of serious problems are caused such as over-nutrition in water bodies, ecological degradation, poor water retention capacity, decrease of biological diversity and variation of river landscape.
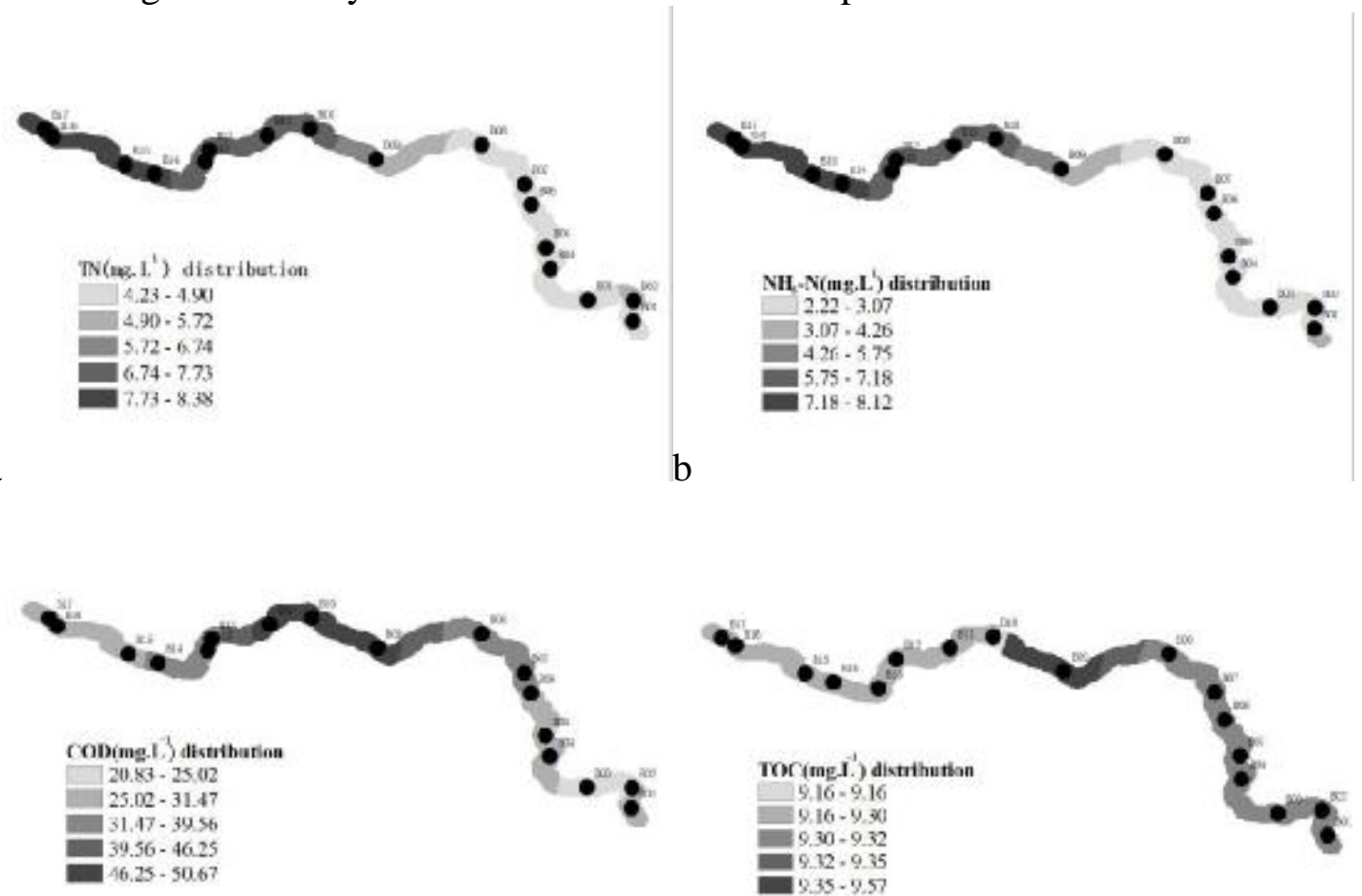

c
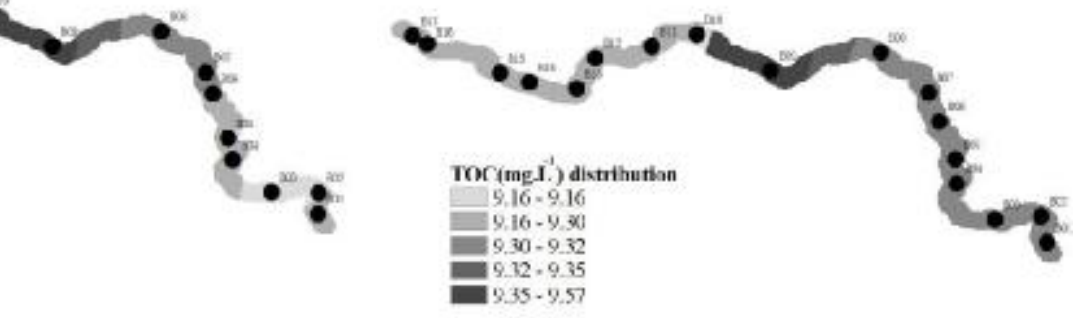

d

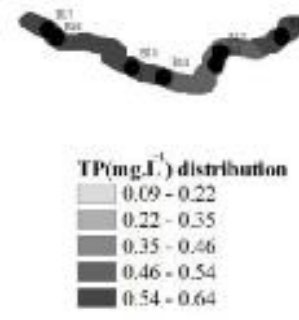

e
(1)

Fig. 2 a-f Spatial distribution of monthly arithmetic mean values of 6 parameters monitored in Baitapu River 
Temporal distribution

Average value and standard errors of means for 6 parameters of different sites collected from Baitapu river( in two years ) were shown in Fig. 3. The variation trend of TOC is most consistent with those of COD, except TOC concentration is higher in winter, which may depict some relevance between them. The accumulation of solid waste pollutants was washed into Baitapu river, which led to a rising of COD and TOC concentration; In addition, significant amounts of agricultural non-point source pollution was washed by melt water, This is another reason of COD and TOC concentration increased. In normal season and wet season, the trends of $\mathrm{TN}$ and $\mathrm{NH}_{3}-\mathrm{N}$ were similar. Because of large runoff, the pollutant concentration was dropped to a lower value. In the other seasons, it changed with the emissions of pollutants along the river. As for chl-a, during April to July, thriving communities of algae can lead a high value. From August to October, though the temperature condition was suitable, with a large quantity of water and low concentrations of TN and TP, algae grew slowly. This may result in the Chl-a concentration lower than usual. Pollutant index concentration change evidently according to the seasons. In summer and fall, phosphorous, nitrogen and organic matter have a lower concentration. That may because pollutants are fully diluted in river. However, in winter and spring, each pollutant index concentration has a substantial increase because of the small amount water.

a

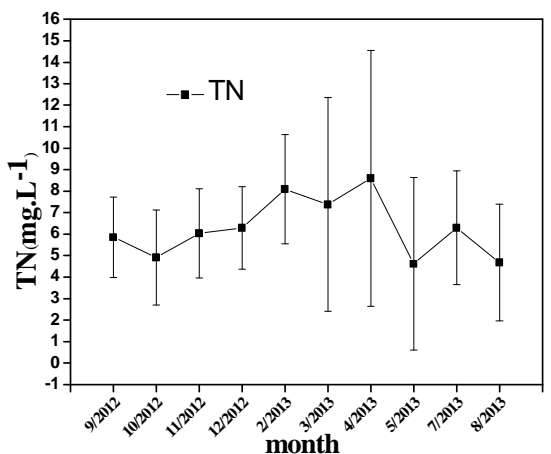

$\mathrm{c}$

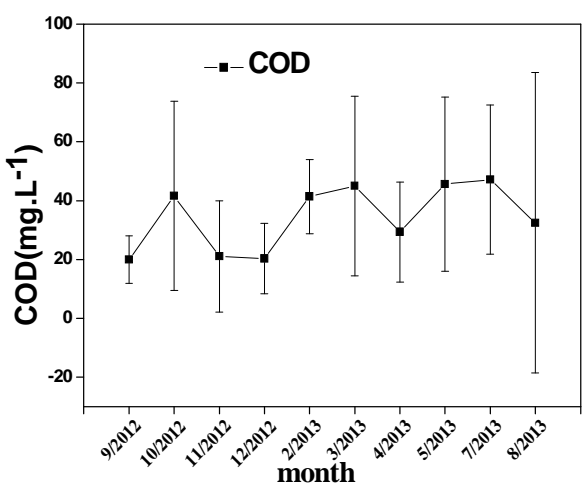

$\mathrm{e}$

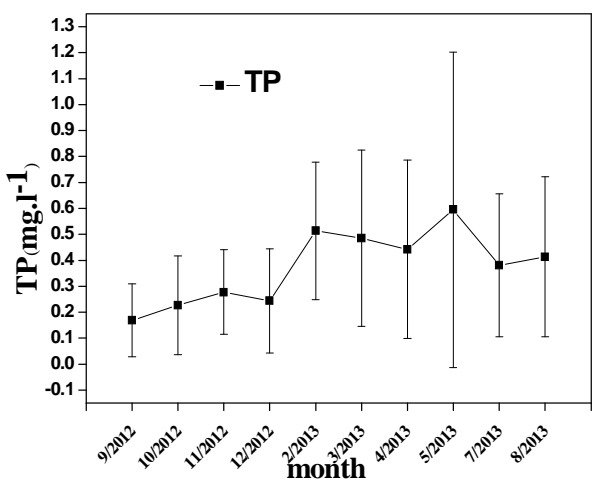

b

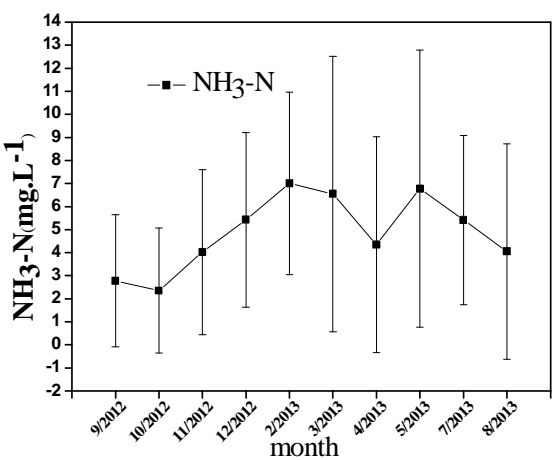

d
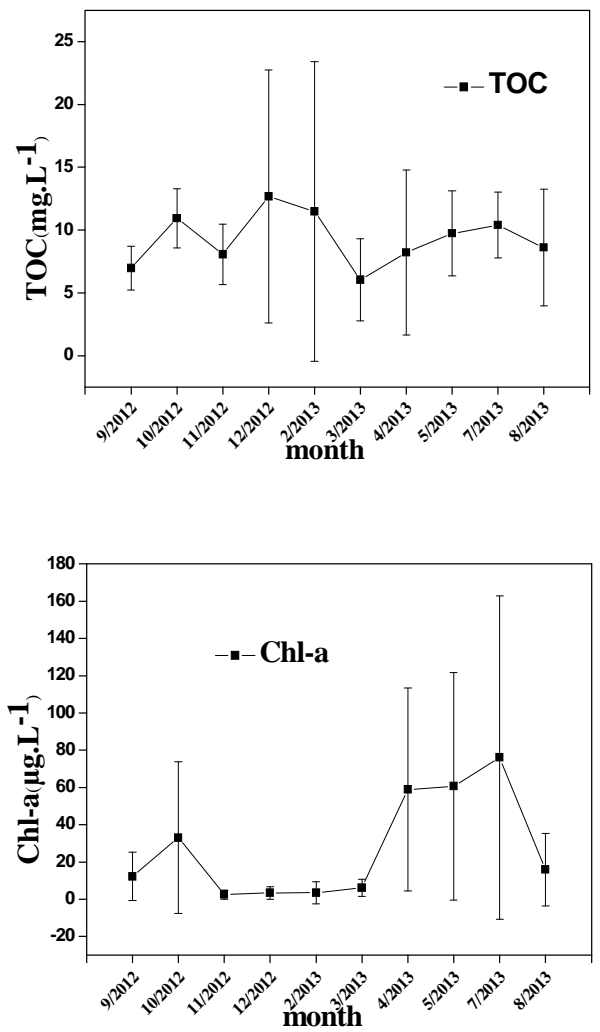

Fig. 3 a-f Temporal distribution of arithmetic mean of 6 parameters monitored in Baitapu River from September 2012 to August 2013 
Principal component analysis of water quality

To examine the availability of the PCA, the Kaiser-Meyer-Olkin(KMO) and Bartlett's sphericity tests were applied to the parameter correlation matrix. Before conducting the PCA, the test results demonstrated that PCA can be used significantly in dimensionality.

According to table 1, the eigenvalues of Component 1,2,3 are respectively 4.921, 2.086, 1.306, which are all over 1 . Meanwhile, total variance (\%) are respectively 49.208\%, 20.862\%, 13.056\%, and the sum of them reaches $83.127 \%$. The evaluation indicator is selected by the variance contribution ratio of the principal component $[8,9]$. According to the principle that sum total variance contribution rate of several principal components should over $80 \%$, when the variance contribution ratio of the first principal component is less than $80 \%$, all the information of indicators should be considered in the first few principal components [8,9]. Component 1 , which explains $49.208 \%$ of the total variance, has strong positive loading on $\mathrm{EC}, \mathrm{TN}, \mathrm{TP}, \mathrm{NH}_{3}-\mathrm{N}, \mathrm{Chl}-\mathrm{a}$ and negative loading on DO. Thus, Component 1 represents that inorganic pollutant is very serious. Component 2 (20.862\% of the total variance) has strong positive loading on PH and COD, reflected organic pollution of water bodies. Component 3 (13.056\% of the total variance) includes ORP and TOC, as component 2, which represents the ion distribution in water. It turns out that the main pollution factors of Baitapu River are $\mathrm{TN}, \mathrm{TP}, \mathrm{NH}_{3}-\mathrm{N}, \mathrm{COD}, \mathrm{TOC}$ and $\mathrm{Chl}-\mathrm{a}$. In these indicators, $\mathrm{TN}, \mathrm{TP}, \mathrm{NH}_{3}-\mathrm{N}$ represented nitrogen and phosphorus pollution, which indicated that the sources of pollution mainly came from agricultural non-point pollution and industrial pollution. COD, TOC and Chl-a represented organic pollution and water eutrophication, mainly came from domestic pollution sources. Typically, agricultural pollution, domestic sewage pollution and industrial pollution are the chief source of pollution for most of north rivers. But actually, it needs a concrete analysis of special rivers by virtue of this method.

Table 1 Eigenvectors and eigenvalues on the correlation matrixes of water quality indexes of Baitapu

\begin{tabular}{cccc}
\multicolumn{4}{c}{ River } \\
\cline { 2 - 4 } Indicators & \multicolumn{3}{c}{ Component } \\
\cline { 2 - 4 } & -0.894 & 0.268 & 0.088 \\
DO & -0.373 & 0.857 & 0.147 \\
PH & -0.320 & 0.144 & 0.821 \\
ORP & 0.936 & -0.091 & 0.174 \\
EC & 0.916 & -0.124 & 0.007 \\
TN & 0.891 & 0.337 & 0.147 \\
TP & 0.963 & -0.017 & 0.056 \\
NH3-N & 0.290 & 0.886 & -0.042 \\
COD & 0.584 & 0.339 & 0.258 \\
Chl-a & 0.139 & 0.507 & -0.692 \\
TOC & 4.921 & 2.086 & 1.306 \\
Eigenvalue & 49.208 & 20.862 & 13.056 \\
Total variance $(\%)$ & 49.208 & 70.071 & 83.127 \\
Cumulative variance $(\%)$ & & &
\end{tabular}

Nemerow index of water quality

Nemerow index was put forward in the book of Scientific Stream Pollution Analysis which was written by professor N. L .Nemerow[10]. TN, TP, $\mathrm{NH}_{3}-\mathrm{N}, \mathrm{COD}$ and $\mathrm{BOD}_{5}$, the five indicators was chosen as evaluation factors. The grading ranges of different Nemerow index represent the corresponding pollution level(Table 2). 
Table 2 Water quality index and contamination levels

\begin{tabular}{cc}
\hline contamination levels & $I_{p}$ \\
\hline Clean & $<0.5$ \\
Still clean & $0.5-1$ \\
Slightly polluted & $1-2$ \\
Moderately polluted & $2-5$ \\
Heavily polluted & $5-10$ \\
Severely polluted & $>10$ \\
\hline
\end{tabular}

Nemerow index of baitapu river(Fig.4) demonstrates an obvious variation trend from B01 to B17. According to the Nemerow index, two categories can be divided for the water quality of Baitapu river. Basically, the first category from B01 to B08 $\left(I_{p}<2\right)$ shows a minor contamination of water quality comprehensive evaluation results. Due to a village located near the river, part of untreated life sewage and solid wastes are directly discharged into the water, which lead to $I_{p}$ of B02 is greater than 2 . The second category, from B09 to B17 $\left(2<I_{p}<5\right)$, presents a moderate pollution. In general, downstream water quality is worse than the upstream in accordance with the degree of pollution.

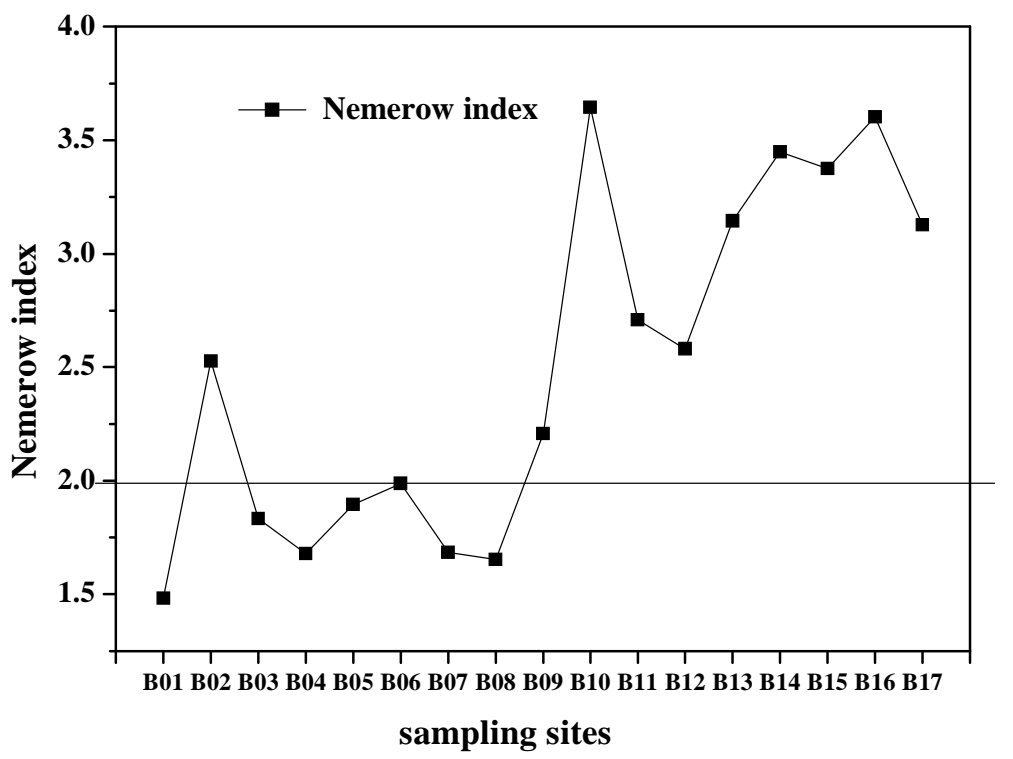

Fig.4 Nemerow index of baitapu river

Source identification

Combined with the spatial variation of water quality indicators and Nemerow index of baitapu river(Fig.5), two sections can be divided for the water quality of Baitapu river. The first segment corresponded to the rural areas and rural-urban fringe zone (B01 - B08), with good water quality indicators and light pollution. Agricultural non-point pollution and dispersed point source pollution are the main source of water pollution in upstream slightly polluted segment. The increasing discharge of domestic wastewater, the loss of chemical fertilizers in agricultural production and the sewage from livestock culture are the significant source of pollution. However, due to the effect of self-purification of the river, pollutants can be degraded in some extent. The second category (B09 B17), located in the urban region, with bad water quality indicators and moderate pollution. In this area, municipal sewage and industrial effluents which are caused by human activities discharged directly into the river. Though parts of the sewage disposal rates is high, higher human-population density and greater amounts of sewage discharge lead to the descending of water body self-purification ability. As a result, water pollution level is more serious than the first category. 


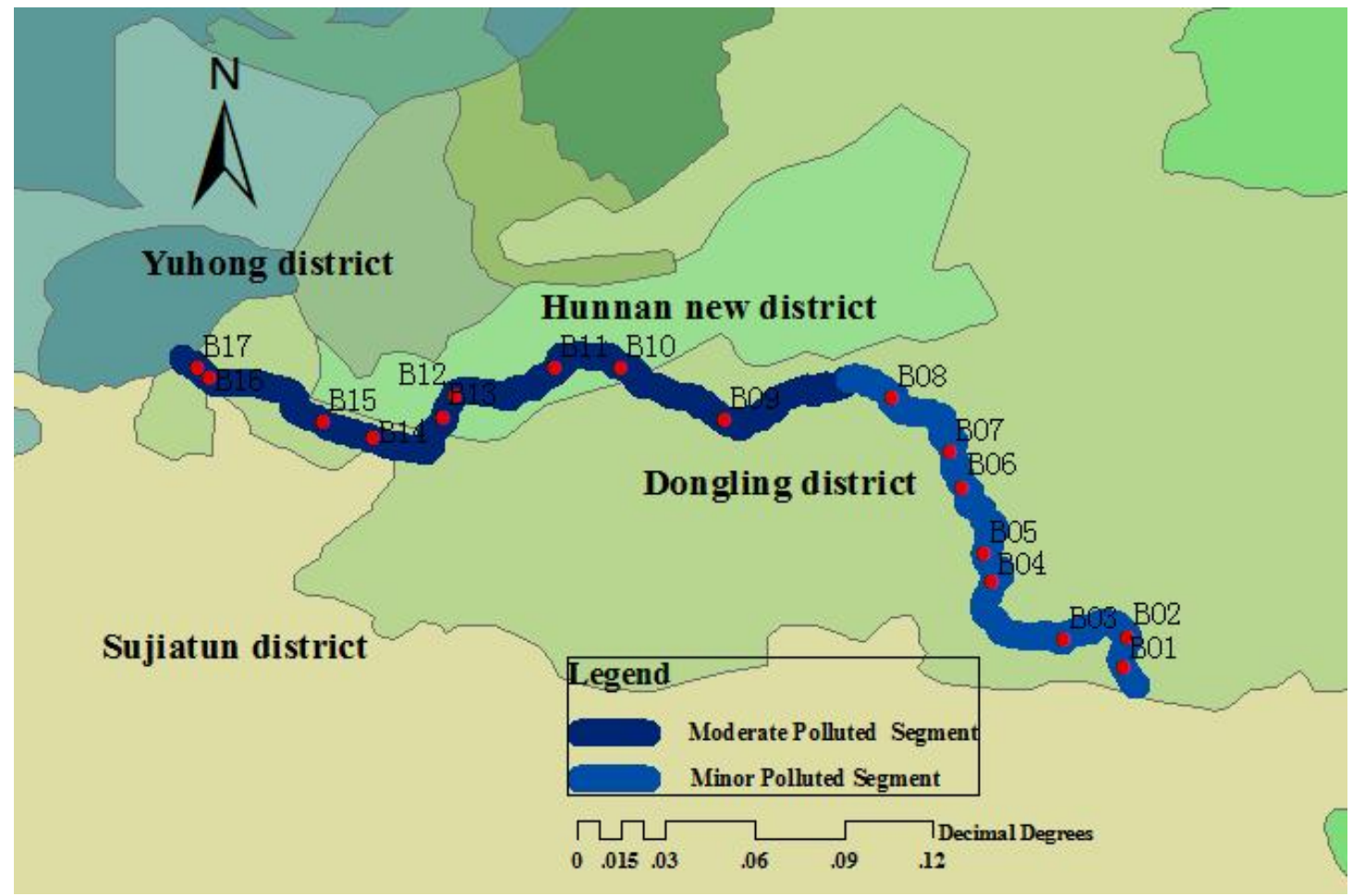

Fig. 5 Spatial differences of Baitapu River

\section{Conclusions}

Kriging interpolation method, PCA and Nemerow index were combined to make an analysis of the the environmental heterogeneity of Baitapu River water quality. From the temporal and spatial differences in water quality, a conclusion can be drawn that $\mathrm{TN}, \mathrm{NH}_{3}-\mathrm{N}$ and $\mathrm{TP}$ pollution are mainly concentrated in the lower reaches and COD, TOC and Chl-a pollution in the middle stream has a higher pollutant concentration. Based on obtained information, it is possible to design a future, optimal sampling strategy, which could reduce the type and pollution degree of northeast rivers. TN, $\mathrm{TP}, \mathrm{NH}_{3}-\mathrm{N}$ pollution mainly came from agro-nonpoint and industrial source pollution, while COD, TOC mainly come from domestic pollution. In addition, Chl-a represented natural algae pollution. The results show that Baitapu river can be dividend into two segments, respectively are upper river lighted pollution segment and downstream middle level pollution segment. Urban river landscape construction and ecological river construction should be paid more special attention. Furthermore, strengthen the processing of industrial, domestic and agricultural non-point source pollution is also urgent affairs. According to this study, interventions should be made to reduce anthropogenic discharges in the river basin and future planning for long-term health of northeast China rivers should be put forward.

\section{Acknowledgements}

This work was financially supported by the Major Science and Technology Program for Water Pollution Control and Treatment of China (2012ZX07202-005).

\section{References}

[1]Lin Ye, Daofeng LI, Tao Tang, Xiaodong Qu and Qinghua Cai: Chinese Journal of Applied Ecology Vol. 14 (2003), p.1959-1962. In Chinese 
[2]Guofeng Li,Xianbin Liu, Zhanguang Liu and Weihua G: Journal of Ecology and Rural Environment Vol. 27 (2011),p.27-31. In Chinese

[3]G. Boren: Environmental Science Vol. 4(1979) ,p.67-71

[4]R.J. Rowley: The Professional Geographer Vol. 63(2011),p.155-157

[5]P.M. Bartier, C.P.Keller: Computer and Geosciences Vol. 22(1996),p.795-799

[6]Cong Xu, Qin Cao and Yuan Yuan: Resource Development and Market Vol. 24(2008) ,p. 118-179. In Chinese.

[7] B.Helena, R.Pardo, M.Vega, E.Barrado, J.M.Fernandez and L.Fernandez: Water Research Vol. 34(2000), p.807-816.

[8]Lihua Feng: Mathematics in practice and theory Vol. 33(2003),p.32-35. In Chinese

[9]Jianhua Chen,Fe Ye: Journal of Gansu Sciences Vol. 15(2003),p.98-100. In Chinese

[10]Bin Xu ,Canyao Lin and Xinwei Mao: Water Resource protection Vol. 2(2014),p.38-40. In Chinese 